\title{
The in vivo effect of L-arginine on skin elasticity in mice
}

\author{
Ádria do Prado Barros de Souza', Marina Miranda Rocha de Oliveira1, Rafael Rocha de Andrade ${ }^{3}$, \\ Rivadávio Fernandes Batista de Amorim³, Anamélia Lonrezetti Bocca², Maria de Fátima Borin ${ }^{1 *}$
}

${ }^{1}$ Faculty of Health Sciences, University of Brasilia, DF, Brazil, ${ }^{2}$ Institute of Biology, University of Brasilia, DF, Brazil, ${ }^{3}$ Faculty of Medicine, University of Brasilia, DF, Brazil

\begin{abstract}
The human skin aging process is a complex mechanism that can be induced both by intrinsic and extrinsic factors. Observations include a decrease in the biosynthetic and proliferative capacity of cells, increased expression of matrix metalloproteinases, reduction in collagen type I expression, and the progressive disappearance of elastic tissue in the papillary dermis. L-arginine, the substrate of nitric oxide synthesis, is involved in angiogenesis and cell proliferation, as well as an indirect precursor of collagen synthesis via the proline pathway. The aim of this study was to examine the tensile strength, histology, and immunohistochemistry of female and male mice skin receiving different concentrations of topically applied L-arginine, in order to evaluate the possibility of using L-arginine as an active cosmetic ingredient in antiaging products. The results suggest that the application of L-arginine improves the mechanical resistance of skin from older female mice ( 20 weeks old) and promotes the formation of a larger amount of collagen and elastic fibers in the skin when applied at a concentration of $15 \%$.
\end{abstract}

Keywords: L-arginine. L-arginine/in vivo efficacy. Skin aging. Elastin. Collagen. iNOS.

\section{INTRODUCTION}

Like all organs, skin undergoes chronological aging, but unlike others, it is in direct contact with the environment, and therefore further ages as a result of environmental damage (Fisher et al., 2002; Pageon et al., 2007; Hwang, Yi, Choi, 2011; Levakov et al., 2012). The aging of human skin is a complex process, induced by both intrinsic factors resulting from tissue degeneration that are largely genetically determined, and by extrinsic factors caused by environmental exposure, predominantly including ultraviolet radiation, smoking, excessive alcohol drinking, and malnutrition. Among the external factors, sun exposure is considered the most harmful to skin (Naylor, Watson, Sherratt, 2011; Jenkins, 2002; Baumann, 2007; Levakov et al., 2012; El-Domyati, Medhat, 2015; Kammeyer, Luiten, 2015).

In intrinsic or chronological aging, changes in the skin are similar to those that occur in most of the internal organs. The changes occur partly because of endogenous injury accumulation due to the continuous formation

\footnotetext{
*Correspondence: M. F. Borin. Laboratório de Farmacologia Molecular, Departamento de Farmácia, Faculdade de Ciências da Saúde, Campus Universitário Darcy Ribeiro, Universidade de Brasília, 70910-900 - Brasília - DF, Brasil. E-mail: mfborin@unb.br
}

of reactive oxygen species (ROS), which are generated by cellular oxidative metabolism (Jenkins, 2002; Tobin, 2017). Although the stratum corneum remains relatively unchanged throughout the aging process, the epidermis and dermis undergo a flattening of the dermal-epidermal junctions and a reduction in the proliferative and biosynthetic capability of skin cells, especially fibroblasts. This results in a decrease in the dermal matrix production and increased expression of matrix metalloproteinases (MMPs) (Jenkins, 2002; Oriá et al., 2003; Langton et al., 2010; Levakov et al., 2012; Tobin, 2017).

The main molecular components involved in the skin aging process are collagen, elastic fibers, and glycosaminoglycans. Among them, collagen is the most abundant extracellular component in skin that imparts the dermis tensile properties (Naylor, Watson, Sherratt, 2011; Jenkins, 2002). Collagen molecules are initially synthesized as a precursor, procollagen, that is posttranslationally hydroxylated by prolyl hydroxylase, generating hydroxyproline residues that are required for the formation of collagen. Together, hydroxyproline and proline residues correspond to $23 \%$ of the collagen molecule (Bellon et al., 1987; Barbul, 2008; Wu et al., 2011; Pokidysheva et al., 2013). Proline used for collagen biosynthesis in fibroblasts may be derived from glutamine, 
glutamate, ornithine, or arginine (Wu et al., 2011).

Previous studies have detailed the relationship between arginine, collagen synthesis, and deposition of collagen in wound-healing processes (Albina, Abate, Mastrofrancesco, 1993; Soneja, Drews, Malinski, 2005). Specifically, research has demonstrated that the proline pool size determines the rate of collagen synthesis, and the local synthesis of proline from their metabolic precursors, such as ornithine, arginine, glutamate and glutamine, is strengthened in some circumstances, apparently to compensate for a relative deficiency in preformed proline residues (Barbul, 2008).

L-arginine is a substrate for nitric oxide synthesis; therefore, it is indirectly involved in many important regulatory mechanisms, such as cell proliferation and angiogenesis (Shi et al., 2003; Durante, 2013). L-arginine is metabolized by nitric oxide synthase (NOS) to nitric oxide (NO) and L-citrulline, or is metabolized to urea and ornithine by arginase- 1 in the liver urea cycle. Ornithine, through the action of ornithine- $\gamma$-aminotransferase is converted to pyrroline-5-carboxylate, that is then metabolized to L-proline by pyrroline-5-carboxylate reductase. L-proline is required for the synthesis of many structural proteins, including collagen (Barbul, 2008; Durante, 2013).

Arginase- 1 has been assumed to participate in wound-healing processes because it affects local cell proliferation and possibly collagen metabolism (Shi et al., 2003). Arginine supplementation in the diet of humans and rodents has a significant effect on the wound-healing process. Rats treated with L-arginine display increased tensile strength of scar skin and higher collagen deposition in comparison with controls (Seifter et al., 1978 apud Soneja, Drews, Malinski, 2005).

Therefore, the objective of this work was to assess the effect of the topical administration of L-arginine on female and male mouse skin elasticity to evaluate the possibility of its use as a cosmetic active ingredient.

\section{MATERIAL AND METHODS}

\section{In vivo efficacy of L-arginine in improving mouse skin elasticity}

To assess the effect of L-arginine on skin elasticity, Swiss female and male mice weighing between 20 and $25 \mathrm{~g}$ each were grouped according to age and sex. Group 1 comprised of female mice at 4-6 weeks old; group 2, female mice at 11-13 weeks old; group 3, female mice at 20 weeks old; group 4, male mice at 4-6 weeks old; group 5 , male mice at 11-13 weeks old; and group 6, male mice at 20 weeks old. The mice groups were housed in plastic cages under controlled light (12 h light/dark cycle) and temperature $\left(25^{\circ} \mathrm{C}\right)$ conditions with water and food $a d$ libitum. To evaluate the in vivo efficacy of L-arginine in improving skin elasticity, the mice received a topical application of $0.5 \mathrm{~g}$ of L-arginine dispersed in glycerol at different concentrations, or glycerol only (vehicle control group, Glyc.) for 15 days, or received no treatment (control group, CTRL). After the treatment period, the mice were euthanized in a carbon dioxide chamber. The abdominal skin of the mice was then shaved and samples of approximately $3 \mathrm{~cm}$ were removed. The fragments of removed tissue were either fixed in buffered formalin and processed for histological evaluation or used immediately to evaluate skin elasticity. All animal procedures were performed according to the rules of CONCEA (The Brazilian National Council for the Control of Animal Experimentation) and carried out under the approval of the Ethics Committee on Animal Use at the University of Brasilia (process number: 47217/2009). All efforts were made to minimize animal suffering.

\section{Skin elasticity evaluation}

The tissue samples that were removed from the mice were kept moist by the addition of saline solution. Skin elasticity was evaluated by measuring tensile strength using VersaTest ${ }^{\circledR}$ equipment coupled with a dynamometer. Skin samples were stretched until rupture and the maximum traction force tolerated by the tissue was determined.

\section{Histological and immunohistochemical evaluation}

Skin fragments from treated mice were immersed in $10 \%$ formaldehyde for $48 \mathrm{~h}$, followed by use in routine histological procedures for paraffin embedding. Histological cross sections were made through the major axis of the skin fragments. Staining techniques used include hematoxylin and eosin (H\&E) staining for the morphological analysis of tissue, Masson's trichrome staining for the analysis of collagen fibers (Hotchkiss, 1948), and Verhoeff's staining for the observation of elastic fibers (Verhoeff, 1908). The sections were examined by light microscopy and analyses were based on the histological characteristics of the tissue in comparison with the control group.

Tissue sections were also immunohistochemically stained using the immunoperoxidase technique in order to analyze inducible NOS (iNOS) expression, as described in the Spring Bioscience and Biogen protocol. The polyclonal antibody rabbit anti-iNOS (Spring Bioscience, 
code E3740), was diluted in antibody diluent (Spring Bioscience, code ADS-125) at ratio of 1:100. The polymer used was Histofine ${ }^{\circledR}$ (Nichirei, Cod. 414341F) and the chromogen substrate was 3,3'-diaminobenzidine (DAB; Spring Bioscience, code DAB-125). Mice lung sections were used for positive and negative controls. For negative control staining, all steps were carried out except for the application of the primary antibody.

The results were expressed as the staining intensity scored with a semiquantitative ordinal scale $(0-30 \%, 30$ $70 \%$, and $70-100 \%$ ) according to analyses by three blinded observers, and the mean was used as the final score.

\section{Statistical Analyses}

The obtained results were statistically analyzed by a variance test (one-way ANOVA) followed by Bonferroni, Dunnett, or Tukey multiple comparisons tests.

\section{RESULTS}

\section{Skin elasticity evaluation}

Skin samples were evaluated from mice that were treated with different concentrations of L-arginine suspended in glycerol, and with glycerol only (Glyc.) or that received no treatment (CTRL) to determine the skin's resistance to mechanical force using the VersaTest ${ }^{\circledR}$ equipment coupled to a dynamometer (Figure 1 and 2).

Figure 1 (A to $\mathrm{C}$ ) presents the results obtained for the evaluation of the mechanical tensile strength of skin from female mice of different ages treated with different concentrations of L-arginine. For mice from group 3 (Figure 1C), treatment with L-arginine at any of the tested concentrations resulted in a significant increase in the tensile strength needed to disrupt the skin. For mice from group 2 (Figure 1B), a significant difference was observed between the control group and the group treated with L-arginine at $10 \%$ that showed an increase in elasticity. The mechanical resistance of skin samples from group 1 mice (Figure 1A) was significantly lower in the control group than in skin from all other groups that were treated with L-arginine or with the vehicle control. Generally, across all groups, there was a trend towards increased mechanical tensile strength of skin treated with L-arginine at $5 \%$ in comparison with controls, and decreased tensile strength for higher concentrations of L-arginine.

Figure 2 shows the results obtained for the treatment of male mice. A significant difference in the tensile force required to rupture the skin of older male mice treated with
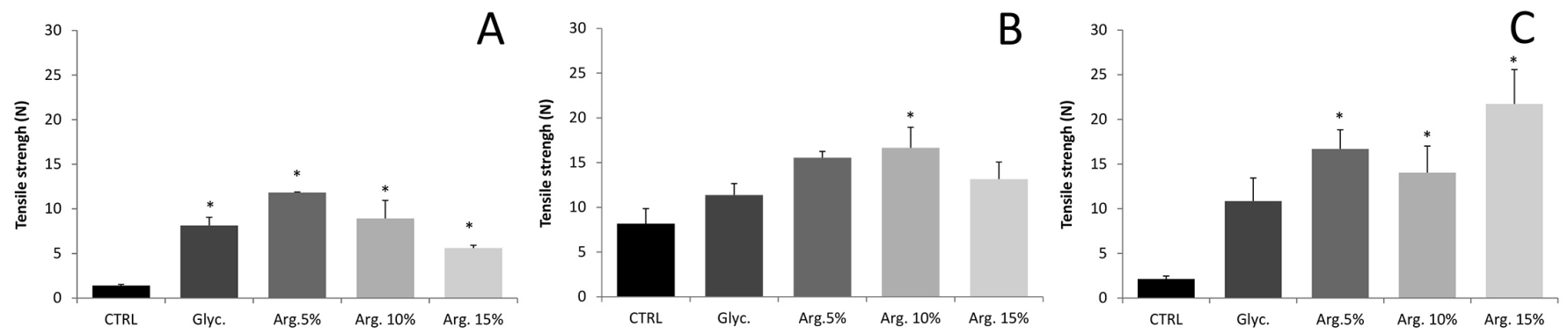

FIGURE 1 - Evaluation of the mechanical tensile strength of female mouse skin. (A) Group 1, (B) group 2, and (C) group 3. The mice were treated with glycerol only (Glyc.), dispersions of L-arginine in glycerol at 5\% (Arg. 5\%), 10\% (Arg. 10\%), or 15\% (Arg. $15 \%$ ), or received no treatment (CTRL). $P<0.05 ;$ * versus $\mathrm{CTRL} ;(3 \leq \mathrm{n} \leq 5)$.
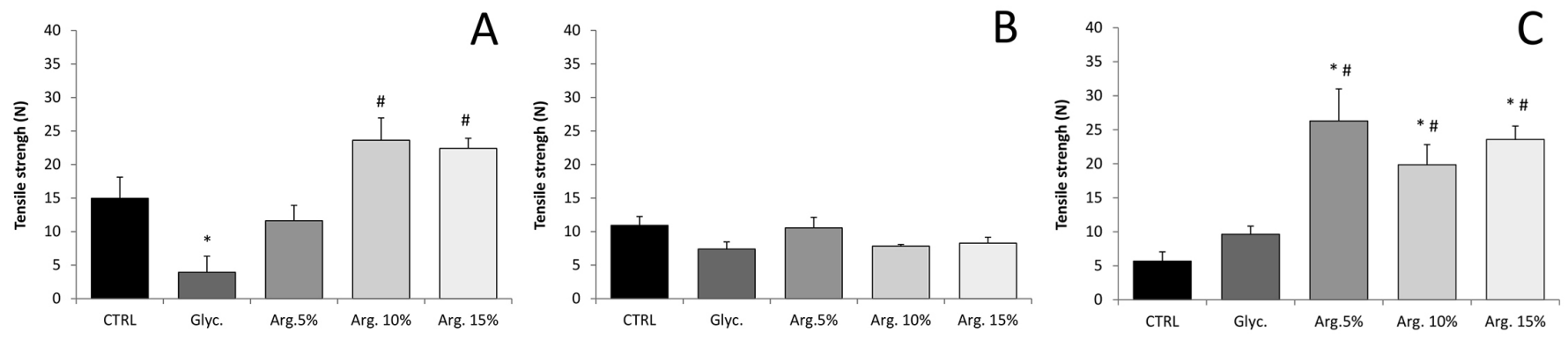

FIGURE 2 - Evaluation of the mechanical tensile strength of male mice skin. (A) Group 4, (B) group 5, and (C) group 6. The mice were treated with glycerol only (Glyc.), dispersions of L-arginine in glycerol at 5\% (Arg. 5\%), 10\% (Arg. 10\%), or 15\% (Arg. $15 \%$ ), or received no treatment (CTRL). $P<0.05$; * versus CTRL, \# versus Glyc.; $(3 \leq \mathrm{n} \leq 5)$. 
L-arginine was observed for all concentrations (Figure 2C). Skin of male mice at 11-13 weeks old displayed no significant difference following treatment (Figure 2B). The experiments performed on the younger male mice revealed that the skin strength of untreated were significantly greater than the skin strength of vehicle control group mice, and significantly lower in the mice of the vehicle group than the groups treated with L-arginine dispersions at concentrations of $10 \%$ or $15 \%$ (Figure $2 \mathrm{~A}$ ).

\section{Histological evaluation}

\section{Evaluation of collagen fibers}

The skin samples underwent routine histological examination and were stained by the Masson's trichrome method to observe collagen fibers. Results were expressed as the staining intensity of fibers scored by three blinded observers, and the mean values for female and male mice are listed in Table I and II, respectively.

TABLE I - Evaluation of collagen fibers stained in female mouse skin

\begin{tabular}{lcc}
\hline Group & & Staining intensity \\
\hline \multirow{3}{*}{ Group 1 } & CTRL & $\mathrm{S}>70 \%$ \\
& Glycerol & $30 \%<\mathrm{S}<70 \%$ \\
& Arg. 5\% & $\mathrm{S}>70 \%$ \\
& Arg. 10\% & $30 \%<\mathrm{S}<70 \%$ \\
& Arg. 15\% & $\mathrm{S}>70 \%$ \\
\hline Group 2 & CTRL & $0<\mathrm{S}<30 \%$ \\
& Glycerol & $30 \%<\mathrm{S}<70 \%$ \\
& Arg. 5\% & $30 \%<\mathrm{S}<70 \%$ \\
Arg. 10\% & $\mathrm{S}>70 \%$ \\
& Arg. 15\% & $0<\mathrm{S}<30 \%$ \\
\hline & CTRL & $30 \%<\mathrm{S}<70 \%$ \\
& Glycerol & $\mathrm{S}>70 \%$ \\
& Arg. 5\% & $30 \%<\mathrm{S}<70 \%$ \\
& Arg. 10\% & $30 \%<\mathrm{S}<70 \%$ \\
\hline
\end{tabular}

The semiquantitative analysis of collagen fibers was performed by three blinded observers. S, mean score of staining.

Table I shows the results for collagen staining of female mouse skin by the Masson's trichrome method. For older mice, L-arginine treatment at higher concentrations produced the best results in comparison with controls, while for younger mice, lower concentrations of L-arginine produced results similar to those for higher concentrations. For male mice (Table II), L-arginine
TABLE II - Evaluation of collagen fibers stained in male mouse skin

\begin{tabular}{lcc}
\hline Group & & Staining intensity \\
\hline \multirow{4}{*}{ Group 4 } & CTRL & $0<\mathrm{S}<30 \%$ \\
& Glycerol & $\mathrm{S}>70 \%$ \\
& Arg. 5\% & $0<\mathrm{S}<30 \%$ \\
& Arg. 10\% & $0<\mathrm{S}<30 \%$ \\
& Arg. 15\% & $\mathrm{S}>70 \%$ \\
\hline \multirow{3}{*}{ Group 5 } & CTRL & $\mathrm{S}>70 \%$ \\
& Glycerol & $0<\mathrm{S}<30 \%$ \\
& Arg. 5\% & $\mathrm{S}>70 \%$ \\
& Arg. 10\% & $\mathrm{S}>70 \%$ \\
Group 6 & Arg. 15\% & $\mathrm{S}>70 \%$ \\
\hline & CTRL & $\mathrm{S}>70 \%$ \\
& Glycerol & $\mathrm{S}>70 \%$ \\
& Arg. 5\% & $0<\mathrm{S}<30 \%$ \\
& Arg. 10\% & $0<\mathrm{S}<30 \%$ \\
\hline
\end{tabular}

The semiquantitative analysis of collagen fibers was performed by three blinded observers. S, mean score of staining.

treatment did not stimulate an increase in collagen fiber abundance. A selection of the photomicrographs that illustrate the analysis are shown in Figures 3 and 4.

\section{Assessment of elastic fibers}

Skin samples were stained by the Verhoeff method for the evaluation of elastic fibers. The obtained photomicrographs were analyzed by counting the fibers in three distinct and random fields of a checkerboard lattice with an area of $0.01 \mathrm{~mm}^{2}$ shared into 100 equal parts (Figure 5). For older mice, the treatment with L-arginine at $15 \%$ resulted in an increase in the amount of elastic fibers in comparison with controls that received the glycerol vehicle only. For younger mice, the treatment did not stimulate any increase in the amount of elastic fibers. The photomicrographs illustrate the difference in the amount of elastic fibers in the skin of younger and older mice, as well as between older control and 15\% L-arginine treated mice (Figure 5).

The difference in the amount of elastic fibers observed among mice of different ages that was observed in female mice was not observed in male mice (Figure 6). Nonetheless, the L-arginine treatment of male mice 11-13 weeks old was effective in stimulating the production of elastic fibers.

Figure 7 depicts the difference in the amount of elastic fibers in untreated skin from male and female 

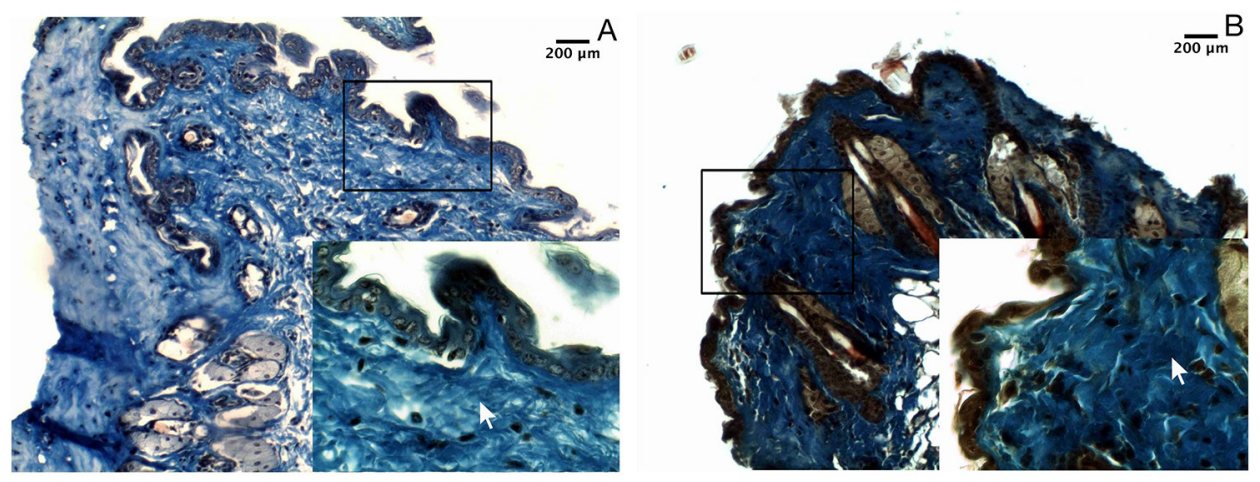

FIGURE 3 - Photomicrographs of skin stained with Masson's trichrome to observe collagen fibers (x100, details x400). (A) Female mouse skin from group 3 treated with vehicle and (B) female mouse skin from group 2 treated with L-arginine 10\%.

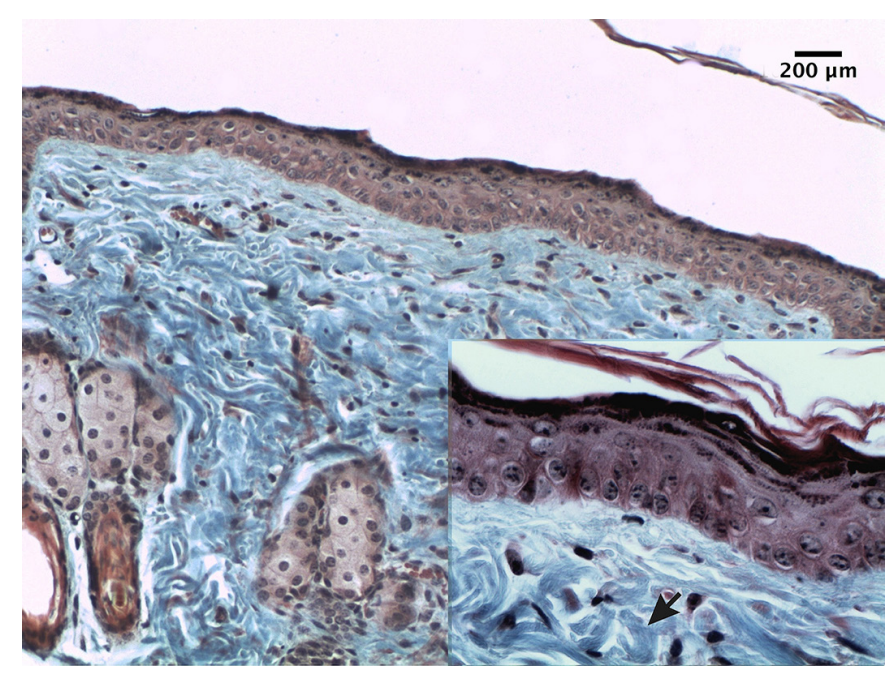

FIGURE 4 - Photomicrograph of male mouse skin from group 6 (CTRL) stained with Masson's trichrome to observe collagen fibers (x100, detail x400).

mice with age. For female control mice, the amount of elastic fibers decreases significantly with aging. This is in contrast with males that maintain a relatively uniform amount of elastic fiber across age groups, despite generally having less elastic fiber in the skin samples than the female mice.

\section{Immunohistochemical detection of iNOS}

The fragments of mouse skin were processed for histological analysis by immunohistochemical staining specific for iNOS detection. Results are expressed as the intensity and distribution of iNOS staining for female (Table III) and male (Table IV) mice.

The mouse skin samples from group 3 stained positive for iNOS, with staining intensity greater than $30 \%$ in the skin appendages for all treatments, including controls (Figure 8A, B, C). A slight positive staining for
iNOS in the epidermis was only observed in CTRL group mice and those treated with L-arginine at 5\% (Figure 8A, B). In group 2, only the skin of female mice that received treatment with L-arginine showed positive staining for iNOS. For group 1, female mice that received treatment with 5\% L-arginine dispersion did not show iNOS staining in the skin.

For male mice, iNOS was not observed in most of the tissue processed by immunohistochemical detection except for in younger mice (group 4). More iNOS was expressed in the skin of older mice treated with $15 \%$ L-arginine (group 6) and in the skin of younger animals treated with 10\% L-arginine (group 4, Table IV). Figure 9 illustrates the photomicrograph of skin from 4-6-week-old mice treated with L-arginine at $10 \%$.

\section{DISCUSSION}

With increasing life expectancy, modern women can spend more than a third of life in the post-menopausal period. During this period, hormonal changes, such as the loss of estrogen production, accelerate changes in the collagen and elastic fibers in skin, resulting in increased wrinkling and sagging after menopause. Research has shown that approximately $30 \%$ of collagen is lost in the first 5 years of this period, and that the rate of collagen loss is $2.1 \%$ per year after menopause (Son et al., 2005, Phuong, Maibach, 2015).

Several substances have been tested to assess their contribution to delaying the skin aging process, by various mechanisms, such as scavenging of ROS and restoration of the redox balance (Hwang, 2010), inhibiting the expression or activity of MMP (Valenti et al., 2011), increasing the expression or regulation of tissue inhibitor of metalloproteinases (TIMP) (Landau, 2007), inhibition of elastosis (Langton et al., 2010), or simply by protecting against ultraviolet (UV) radiation that promotes the 

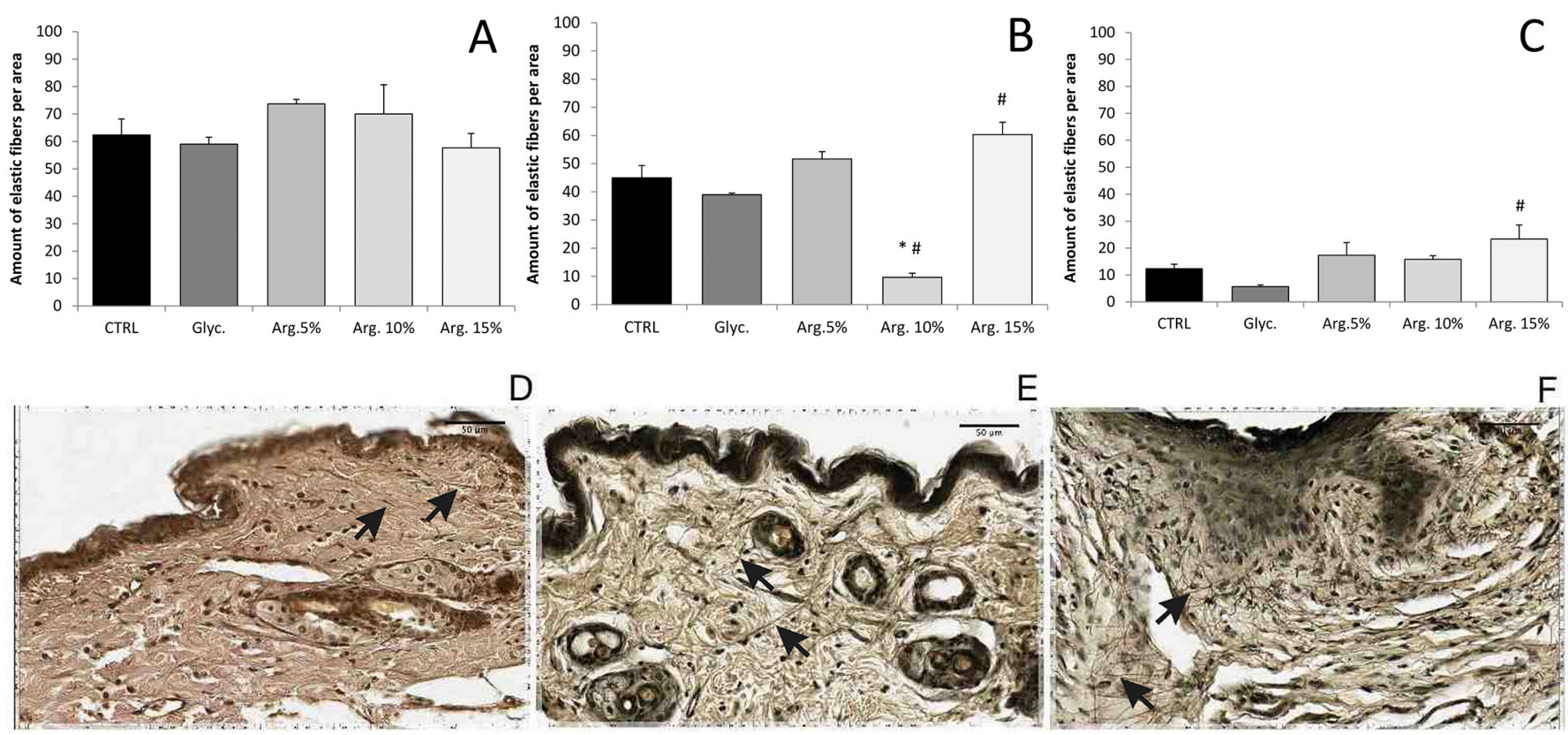

$E$

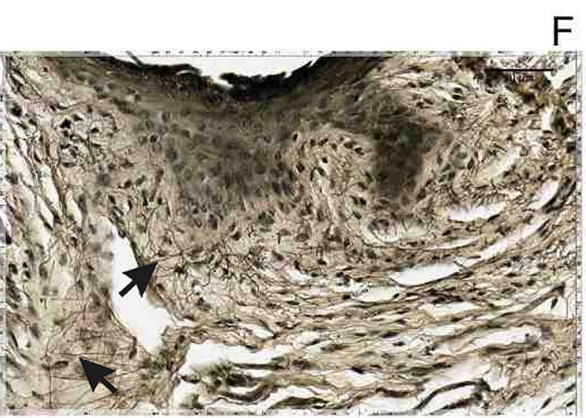

FIGURE 5 - Quantitative analysis of elastic fibers in female mouse skin. Mice from group 1 (A), group 2 (B), and group 3 (C) were treated with vehicle (Glyc.), L-arginine at 5\% (Arg. 5\%), 10\% (Arg. 10\%), or 15\% (Arg. 15\%), or did not receive any treatment (CTRL). $P<0.05$, * versus CTRL, \# versus Glyc.; $(3 \leq \mathrm{n} \leq 5)$. (D-F) show the photomicrographs (x400) of the skin sections stained for evidence of elastic fibers using the Verhoeff method: (D), mice from group 3, CTRL; (E), mice from group 3 treated with L-arginine at 15\%; and (F), mice from group 1 treated with L-arginine at 10\%.
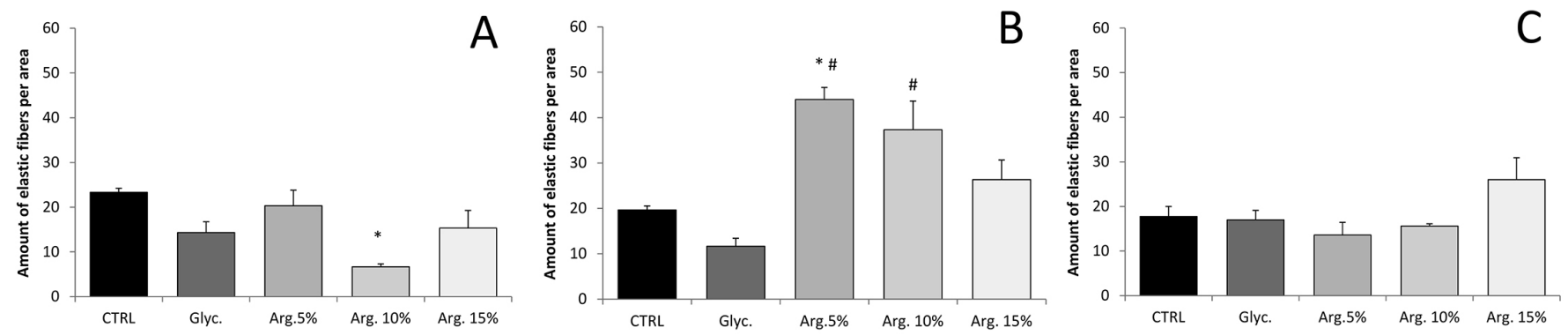

FIGURE 6 - Quantitative analysis of elastic fibers in male mouse skin. Mice from group 4 (A), group 5 (B), and group 6 (C) were treated with vehicle (Glyc.), L-arginine at 5\% (Arg. 5\%), 10\% (Arg. 10\%), or 15\% (Arg. 15\%), or did not receive any treatment (CTRL). $P<0.05$, * versus CTRL, \# versus Glyc.; $(3 \leq \mathrm{n} \leq 5)$.

formation of ROS and triggers many of these processes that are responsible for aging (Choe et al., 2003).

As already mentioned, Seifter and collaborators (Soneja, Drews, Malinski, 2005) described an increase in tensile strength from scar and collagen deposition in the healing process in rats fed the arginine-supplemented diet when compared with that in the control group, fed the arginine-free diet. In the present study, an increased resistance of the skin to traction force found when L-arginine dispersions were topically administered in not only groups 2 and 3 mice, but also in group 1. Collagen fiber analysis revealed an increase in the amount of fibers in the skin of mice from groups that received treatment with higher concentrations of L-arginine in comparison with that at lower concentrations. Estrogen deficiency may be responsible for skin aging at the start of the infertile period, since it affects the loss of collagen and skin water content (Calleja-Agius, Brincat, 2009; Phuong, Maibach, 2015), and it could explain the observed decrease in traction resistance and collagen in the skin of the older group of female mice (group 3). Once L-arginine is metabolized in the urea cycle, yielding ornithine that is used for proline production, which in turn is used for fibroblast collagen synthesis (Barbul, 2008), the amino acid may be contributing to the increase of elasticity and the amount of collagen fibers in the skin of treated mice. 


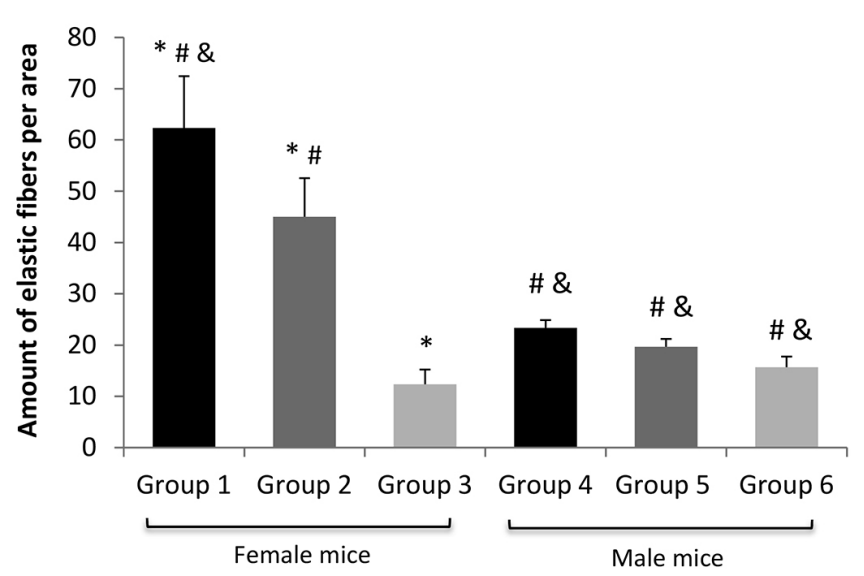

FIGURE 7 - Amount of elastic fibers in the skin of male and female mice. The mice did not receive any kind of treatment (CTRL). Elastic fibers were counted in three distinct and random fields of a checkerboard lattice with an area of $0.01 \mathrm{~mm}^{2}$ shared into 100 equal parts. *, \#, and \&, indicate a significant difference between flagged groups, $P<0,05 ; \mathrm{n}=3$.

The histological sections of skin stained with Masson's trichrome for collagen analysis revealed that the male mice from the CTRL group presented more collagen fibers in the skin than the female mice from the CTRL group. This feature was observed for all age groups, except for the youngest mice that had a high intensity of collagen fiber staining in the skin for both male and female mice. This age-dependent difference observed in the skin of females may be explained by estrogen deficiency that is characteristic of the final phases of childbearing age.

L-arginine can also be metabolized in the nitric oxide synthase cycle resulting in NO production. Some studies have shown that NO induces both expression and activation of MMP-1 in chondrocyte cultures, as well as induces MMP-1 and MMP-2 expression and activity in fibroblasts (Lin et al., 2003; Choe et al., 2003). Therefore, iNOS expression in mouse skin was evaluated by immunohistochemistry and the results show an agreement with this supposition, as the skin from group 1 mice treated with vehicle or $10 \% \mathrm{~L}$-arginine had a lower amount of collagen fibers than skin treated with $15 \%$ L-arginine, which also did not exhibit iNOS expression in the epithelium or showed very little iNOS expression in the connective tissue. It is reasonable to assume that $10 \% \mathrm{~L}$-arginine could induce NO production that leads to the induction of collagenase expression. Although several studies indicate that the synthesis of collagen can be inhibited by NO in several types of cells, such as arteriole, vascular smooth muscle, and mesothelial cells (Chatziantoniou et al., 1998; Myers, Tanner, 1998; Owens, Milligan, Grisham, 1996), other studies have shown that NO induces collagen synthesis in fibroblasts in the lamina

TABLE III - Immunohistochemical iNOS staining of female mouse skin

\begin{tabular}{|c|c|c|c|c|}
\hline Group & & Epithelium & Cutaneous annexes & Connective tissue \\
\hline & CTRL & $0<\mathrm{IS}<30 \%, \mathrm{U}$ & $30 \%<\mathrm{IS}<70 \%, \mathrm{U}$ & $30 \%<\mathrm{IS}<70 \%, \mathrm{U}$ \\
\hline & Glycerol & $0<\mathrm{IS}<30 \%, \mathrm{D}$ & $0<\mathrm{IS}<30 \%, \mathrm{D}$ & - \\
\hline \multirow[t]{5}{*}{ Group 1} & Arg. $5 \%$ & - & - & - \\
\hline & Arg. $10 \%$ & IS $>70 \%, \mathrm{U}$ & $30 \%<\mathrm{IS}<70 \%, \mathrm{U}$ & IS > 70\%, U \\
\hline & Arg. $15 \%$ & - & $0<$ IS $<30 \%$, D & $0<\mathrm{IS}<30 \%, \mathrm{D}$ \\
\hline & CTRL & - & - & - \\
\hline & Glycerol & - & - & - \\
\hline \multirow[t]{5}{*}{ Group 2} & Arg. $5 \%$ & $*$ & * & $*$ \\
\hline & Arg. $10 \%$ & $30 \%<\mathrm{IS}<70 \%, \mathrm{U}$ & $0<\mathrm{IS}<30 \%, \mathrm{U}$ & $0<\mathrm{IS}<30 \%, \mathrm{D}$ \\
\hline & Arg. $15 \%$ & $30 \%<\mathrm{IS}<70 \%, \mathrm{U}$ & $30 \%<\mathrm{IS}<70 \%, \mathrm{U}$ & $0<\mathrm{IS}<30 \%$, D \\
\hline & CTRL & $0<\mathrm{IS}<30 \%, \mathrm{D}$ & $30 \%<\mathrm{IS}<70 \%, \mathrm{U}$ & $30 \%<\mathrm{IS}<70 \%, \mathrm{D}$ \\
\hline & Glycerol & - & $30 \%<\mathrm{IS}<70 \%, \mathrm{U}$ & - \\
\hline \multirow[t]{3}{*}{ Group 3} & Arg. $5 \%$ & $30 \%<\mathrm{IS}<70 \%, \mathrm{U}$ & $30 \%<\mathrm{IS}<70 \%, \mathrm{U}$ & $0<\mathrm{IS}<30 \%, \mathrm{D}$ \\
\hline & Arg. $10 \%$ & - & $30 \%<\mathrm{IS}<70 \%, \mathrm{U}$ & - \\
\hline & Arg. $15 \%$ & - & $30 \%<\mathrm{IS}<70 \%, \mathrm{U}$ & - \\
\hline
\end{tabular}

The intensity and distribution of marked iNOS in epithelial skin, cutaneous annexes, and connective tissue are listed. The values are the average of semiquantitative analyses made by three blinded observers. IS, immunohistochemical staining; -, absence of staining; *, insufficient material for analysis. The staining distribution was classified as diffuse (D), focused (F), or uniform (U). 
TABLE IV - Immunohistochemical iNOS staining of male mouse skin

\begin{tabular}{|c|c|c|c|c|}
\hline Group & & Epithelium & Cutaneous annexes & Connective tissue \\
\hline \multirow{5}{*}{ Group 4} & CTRL & $0<\mathrm{IS}<30 \%, \mathrm{D}$ & $30 \%<\mathrm{IS}<70 \%, \mathrm{U}$ & IS $>70 \%, \mathrm{U}$ \\
\hline & Glycerol & $0<\mathrm{IS}<30 \%$, D & $0<\mathrm{IS}<30 \%, \mathrm{D}$ & $0<\mathrm{IS}<30 \%, \mathrm{U}$ \\
\hline & Arg. $5 \%$ & $0<$ IS $<30 \%$, D & $0<\mathrm{IS}<30 \%, \mathrm{D}$ & $0<\mathrm{IS}<30 \%, \mathrm{D}$ \\
\hline & Arg. $10 \%$ & IS > 70\%, U & $30 \%<\mathrm{IS}<70 \%, \mathrm{U}$ & $30 \%<\mathrm{IS}<70 \%, \mathrm{U}$ \\
\hline & Arg. $15 \%$ & - & $0<\mathrm{IS}<30 \%, \mathrm{D}$ & - \\
\hline \multirow{5}{*}{ Group 5} & CTRL & - & - & - \\
\hline & Glycerol & - & - & - \\
\hline & Arg. $5 \%$ & - & - & - \\
\hline & Arg. $10 \%$ & - & - & - \\
\hline & Arg. $15 \%$ & - & $0<\mathrm{IS}<30 \%$, D & - \\
\hline \multirow{5}{*}{ Group 6} & CTRL & - & $0<\mathrm{IS}<30 \%, \mathrm{~F}$ & - \\
\hline & Glycerol & - & $0<\mathrm{IS}<30 \%, \mathrm{~F}$ & - \\
\hline & Arg. $5 \%$ & - & - & - \\
\hline & Arg. $10 \%$ & - & - & - \\
\hline & Arg. $15 \%$ & IS $>70 \%, U$ & IS $>70 \%, U$ & $0<\mathrm{IS}<30 \%, \mathrm{U}$ \\
\hline
\end{tabular}

The intensity and distribution of marked iNOS in epithelial skin, cutaneous annexes, and connective tissue are listed. The values are the average of semiquantitative analyses made by three blinded observers. IS, immunohistochemical staining; -, absence of staining. The staining distribution was classified as diffuse (D), focused (F), or uniform (U).
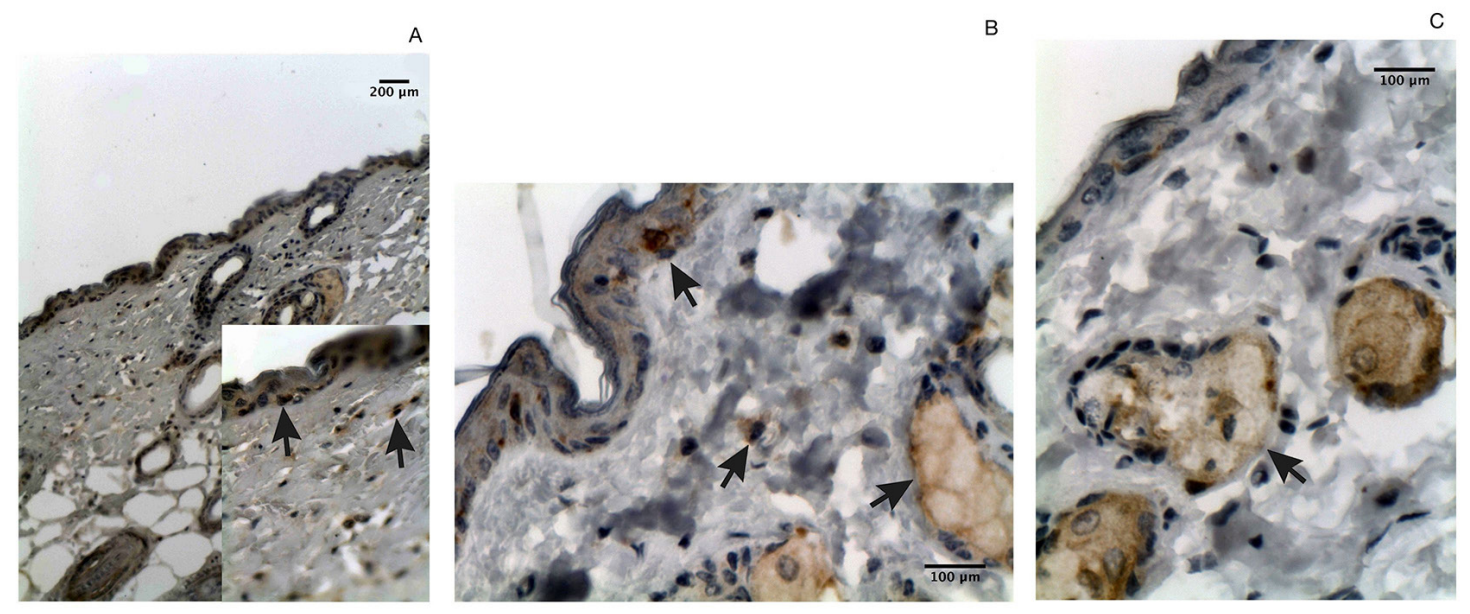

FIGURE 8 - Photomicrographs of immunohistochemical iNOS detection in female mouse skin. Immunohistochemical iNOS detection of group 3 mice. (A) control group, with positive staining in the connective tissue and skin appendages (x100, detail $\mathrm{x} 400$ ); (B) group treated with $\%$ L-arginine, showing positive staining in the epithelial cells, connective tissue, and skin appendages (x400); (C) group treated with L-arginine $15 \%$, with positive staining only in cutaneous annexes (x400).

propria of the small intestine (Chakravortty, Kumar, 1997) and in the re-epithelialization of normal skin as part of the healing processes (Stallmeyer et al., 1999; Hsu et al., 2006).

Witte and coworkers (2000) revealed that although the NO donor used in the study presented a cytostatic effect on fibroblasts, the administration of low concentrations of NO donor in rat dermal fibroblast cultures enhanced collagen synthesis. The authors suggested that NO upregulates collagen synthesis in dermal fibroblasts without affecting collagen breakdown activity, acting by a posttranslational mechanism. L-arginine is a substrate either for arginase or for nitric oxide synthase. Arginase expression regulates arginine bioavailability for $\mathrm{NO}$ synthesis (Gobert et al., 2000; Kavalukas et al., 2012), and despite the $V_{\max }$ of arginase for arginine exceeding that 


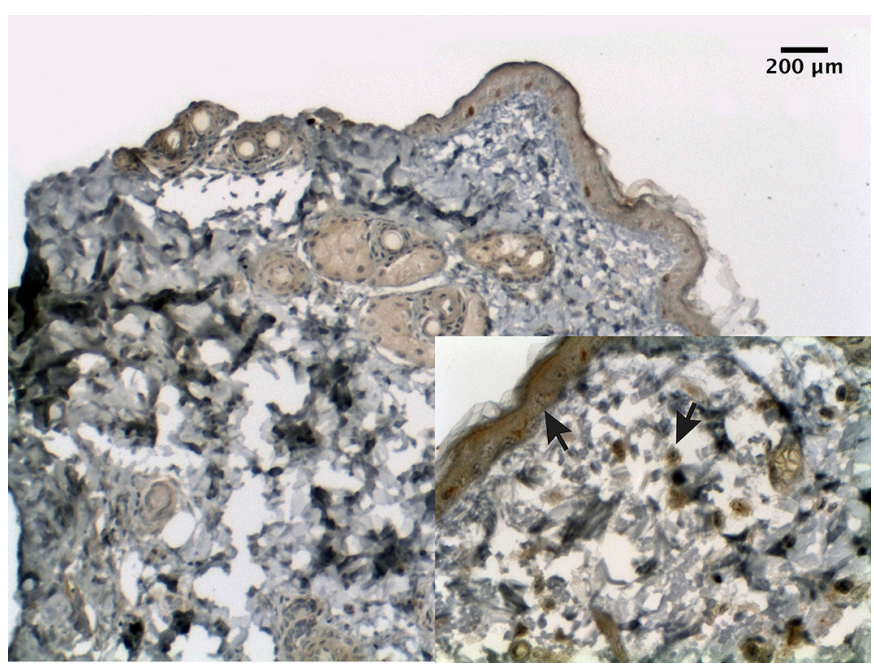

FIGURE 9 - Photomicrograph of immunohistochemical iNOS detection in male mouse skin. Skin of group 4 mice treated with $10 \% \mathrm{~L}$-arginine marked by positive immunohistochemical iNOS staining in the epithelial cells, connective tissue, and cutaneous annexes (x100, detail x400).

of NOS by approximately 1000-fold, the affinity of NOS for arginine is greater (Kavalukas et al., 2012). Thus, in agreement with the results by Witte and coworkers (2000), the results of the present study also suggest that topically administrated arginine can penetrate into the skin, and owing to its higher affinity for NOS, it binds preferentially to NOS in the synthesis of NO, and thereby induces the synthesis of collagen.

Elastic fibers also undergo age-related changes. The fragmentation of elastic fibers results in a decrease in the physiological elasticity of the skin (Waller, Maibach, 2006). Furthermore, aged cells show a reduced ability to resynthesize elastic fibers that have degraded naturally (Jenkins, 2002). In the present study, a significant decrease in the amount of elastic fibers was found with aging in female mice, possibly explained by the estrogen deficiency characteristic of the final stages of childbearing age. Group 3 mice showed the largest amount of elastic fibers in the skin after treatment with L-arginine at $15 \%$ in comparison with mice from other groups. This suggests that L-arginine might contribute to an increase in the amount of elastic fibers in the skin of mice over 20 weeks of age, representing animals in the final stages of reproductive aging.

\section{CONCLUSION}

In the present study, topical treatment with L-arginine improved skin resistance to tensile force in 20-week-old female mice (group 3), as well as treatment with $15 \%$
L-arginine stimulated formation of larger amounts of elastic fibers and collagen. According to these results, L-arginine, which exhibits a hydrophilic character, could be a promising active ingredient in cosmetics to contribute to the postponement of skin aging effects, especially for women nearing the menopausal period.

\section{ACKNOWLEDGEMENTS}

The authors would like to acknowledge the financial support from the Foundation for Research Support from Federal District (FAPDF; Fundação de Apoio à Pesquisa do Distrito Federal), Coordination for the Improvement of Higher Education Personnel (CAPES; Coordenação de Aperfeiçoamento de Pessoal de Nivel Superior), and Brazilian National Council for Scientific and Technological Development (CNPq; Conselho Nacional de Desenvolvimento Cientifico e Tecnológico). The authors would also like to express their gratitude to the Molecular Pharmacology Laboratory and Oral Histopathology Laboratory at the University of Brasilia, and a special thanks to Dr. Eliete Neves da Silva Guerra and Dr. Nilce Santos de Melo for assistance with the histological analysis. The authors state that there is no conflict of interest.

\section{REFERENCES}

Albina JE, Abate JA, Mastrofrancesco B. Role of ornithine as a proline precursor in healing wounds. J Surg Res. 1993;55(1):97102.

Barbul A. Proline precursors to sustain mammalian collagen synthesis. J Nutr. 2008;(138):S2021-S2024.

Baumann L. Skin ageing and its treatment. J Pathol. 2007;211(2):241-251.

Bellon G, Monboisse JC, Randoux A, Borel JP. Effects of preformed proline and proline amino acid precursors (including glutamine) on collagen synthesis in human fibroblast cultures. Biochim Biophys Acta. 1987;930(1):39-47.

Calleja-Agius J, Brincat MP. Effects of hormone replacement therapy on connective tissue: why is this important? Best Pract Res Clin Obstet Gynaecol. 2009;23(1):121-127.

Chakravortty D, Kumar KN. Induction of cell proliferation and collagen synthesis in human small intestinal lamina propria fibroblasts by lipopolysaccharide: possible involvement of nitric oxide. Biochem Biophys Res Commun. 1997;240(2):458-463. 
Chatziantoniou C, Boffa JJ, Ardaillou R, Dussaule JC. Nitric oxide inhibition induces early activation of type I collagen gene in renal resistance vessels and glomeruli in transgenic mice. Role of endothelin. J Clin Invest. 1998;101(12):2780.

Choe T, Lee B, Park I, Hong S. Inhibition of matrix metalloproteinase-1 and -2 expression using nitric oxide synthase inhibitors in UV-irradiated human dermal fibroblasts. J Cosmet Sci. 2003;54(3):229-238.

Durante W. Role of arginase in vessel wall remodeling. Front Immunol. 2013;4:1-12.

El-Domyati M, Medhat W. Skin aging: an immunohistochemical evaluation. In: Textbook of Aging Skin. 2015; 2nd Edition, Springer-Verlag Berlin Heidelberg, 1-17.

Fisher GJ, Kang S, Varani J, Csorgo ZB, Wan Y, Datta S, et al. Mechanisms of photoaging and chronological skin aging. Arch Dermatol. 2002;138(11):1462-1470.

Gobert AP, Daulouede S, Lepoivre M, Boucher JL, Bouteille B, Buguet A, et al. L-Arginine availability modulates local nitric oxide production and parasite killing in experimental trypanosomiasis. Infect Immun. 2000;68(8):4653-7.

Hotchkiss RD. A microchemical reaction resulting in the standing of polysaccaride structures in fixed tissue preparations. Arch Biochem. 1948;16(1):131-141.

Hsu HC, Hsiao M, Wang LF, Chien YW, Lee WR. Nitric oxide produced by iNOS is associated with collagen synthesis in keloid scar formation. Nitric Oxide. 2006;14(4):327-334.

Hwang HJ. Skin elasticity and sea polyphenols. Seanol Sci Center Rev. 2010;1(1):1-10.

Hwang KA, Yi BR, Choi KC. Molecular mechanisms and in vivo mouse models of skin aging associated with dermal matrix alterations. Lab Anim Res. 2011;27(1):1-8.

Jenkins G. Molecular mechanisms of skin ageing. Mech Ageing Dev. 2002;123(7):801-810.

Kammeyer A, Luiten R. Oxidation events and skin aging. Ageing Res Rev. 2015;21:16-29.

Kavalukas SL, Uzgare AR, Bivalacqua TJ, Barbul A. Arginase inhibition promotes wound healing in mice. Surgery. 2012;151(2):287-295.
Landau M. Exogenous factors in skin aging. Curr Probl Dermatol. 2007;35:1-13.

Langton AK, Sherratt MJ, Griffiths CEM, Watson REB. A new wrinkle on old skin: the role of elastic fibres in skin ageing. Int J Cosmetc Sci. 2010;32(5):330-339.

Levakov A, Vuckovic N, Dolai M, Mocko Kacanski M, Bozanic S. Age-related skin changes. Med Pregl. 2012;65(5-6):191-195.

Lin SK, Kok SH, Kuo MYP, Lee MS, Wang CC, Lan WH, et al. Nitric oxide promotes infectious bone resorption by enhancing cytokine-stimulated interstitial collagenase synthesis in osteoblasts. J Bone Miner Res. 2003;18(1):39-46.

Myers PR, Tanner MA. Vascular endothelial cell regulation of extracellular matrix collagen role of nitric oxide. Arterioscl Throm Vas. 1998;18(5):717-722.

Naylor EC, Watson REB, Sherratt MJ. Molecular aspects of skin ageing. Maturitas. 2011;69(3):249-256.

Oriá RB, Ferreira FVA, Santana EN, Fernandes MR, Brito GAC. Estudo das alterações relacionadas com a idade na pele humana, utilizando métodos de histo-morfometria e autofluorescência. An Bras Dermatol. 2003;78(4):425-434.

Owens MW, Milligan SA, Grisham MB. Inhibition of pleural mesothelial cell collagen synthesis by nitric oxide. Free Radical Bio Med. 1996;21(5):601-607.

Pageon H, Bakala H, Monnier VM, Asselineau D. Collagen glycation triggers the formation of aged skin in vitro. Eur $\mathrm{J}$ Dermatol. 2007;17(1):12-20.

Phuong C, Maibach HI. Biological effects of estrogen on skin. Textbook of Aging Skin. 2015:1-12.

Pokidysheva E, Zientek KD, Ishikawa Y, Mizuno K, Vranka JA, Montgomery NT, et al. Posttranslational modifications in type i collagen from different tissues extracted from wild type and Prolyl 3-Hydroxylase 1 null mice. J Biol Chem. 2013;288(34):24742-52.

Shi HP, Most D, Efron DT, Witte MB, Barbul A. Supplemental L-arginine enhances wound healing in diabetic rats. Wound Repair Regen. 2003;11(3):198-203. 
Son ED, Lee JY, Lee S, Kim MS, Lee BG, Chang IS, et al. Topical application of $17 \beta$-estradiol increases extracellular matrix protein synthesis by stimulating TGF- $\beta$ signaling in aged human skin in vivo. J Invest Dermatol. 2005;124(6):1149-1161.

Soneja A, Drews M, Malinski T. Role of nitric oxide, nitroxidative and oxidative stress in wound healing. Pharmacol Rep. 2005;57:108-119.

Stallmeyer B, KaÈmpfer H, Kolb N, Pfeilschifter J, Frank $\mathrm{S}$. The function of nitric oxide in wound repair: inhibition of inducible nitric oxide-synthase severely impairs wound reepithelialization. J Invest Dermatol. 1999;113(6):1090-1098.

Tobin DJ. Introduction to skin aging. J Tissue Viability. 2017;26(1):37-46.

Valenti DMZ, Silva J, Teodoro WR, Velosa AP, Mello SBV. Effect of topical clay application on the synthesis of collagen in skin: an experimental study. Clin Exp Dermatol. 2011;37(2):164-168.
Verhoeff F. Some new staining methods of wide applicability.: including a rapid differential stain for elastic tissue. J Amer Med Assoc. 1908;50(11):876-877.

Waller JM, Maibach HI. Age and skin structure and function, a quantitative approach (II): protein, glycosaminoglycan, water, and lipid content and structure. Skin Res Technol. 2006;12(3):145-154.

Witte MB, Thornton, FJ, Efron DT, Barbul, A. Enhancement of fibroblast collagen synthesis by nitric oxide. Nitr Oxide Biol Chem. 2000;4(6):572-582.

Wu G, Bazer FW, Burghardt RC, Johnson GA, Kim SW, Knabe DA, et al. Proline and hydroxyproline metabolism: implications for animal and human nutrition. Amino acids. 2011;40(4):105363.

Received for publication on $10^{\text {th }}$ October 2016 Accepted for publication on $09^{\text {th }}$ May 2017 